\title{
Estudio comparativo de diferentes procedimientos de sling suburetral para el tratamiento de la incontinencia urinaria de esfuerzo
}

\author{
A. Borrell Palanca, F. Chicote Pérez, J.F. Beltrán Meseguer, J.A. Queipo Zaragozá, J. Esteve \\ Claramunt, F. Pastor Sempere
}

Servicio de Urología. Hospital de Sagunt. Valencia.

Actas Urol Esp 2005; 29 (8): 757-763

RESUMEN

ESTUDIO COMPARATIVO DE DIFERENTES PROCEDIMIENTOS DE SLING SUBURETRAL PARA EL TRATAMIENTO DE LA INCONTINENCIA URINARIA DE ESFUERZO

Objetivos: Comparar los resultados de los diferentes procedimientos de sling suburetral utilizados en nuestro servicio (TVT ${ }^{\circledR}$, Sparc $^{\circledR}$, IVS $^{\circledR}$, Obtape $^{\circledR}$ y Monarc $^{\circledR}$ ) para el tratamiento de la IUE femenina.

Material y métodos: Análisis retrospectivo de 96 pacientes afectas de IUE y sometidas a diferentes procedimientos de sling suburetral sin tensión. La edad media: 56,1 $\pm 10,7$ años. 75 pacientes se intervinieron mediante procedimientos suprapúbicos (48 TVT, 17 Sparc, 10 IVS) y 21 con transobturatrices (10 Obtape, 11 Monarc). El tiempo medio de seguimiento: $26,3 \pm 12,7$ meses (29,4 meses para los métodos suprapúbicos y 11,7 para los transobturarices). Las variables analizadas fueron: edad, obesidad, grado de incontinencia previa, tipo de procedimiento empleado, tiempo de seguimiento, y procedimientos quirúrgicos previos. Comparamos las complicaciones y los resultados obtenidos con las diferentes técnicas.

Resultados: el porcentaje de curación fue del 95,7\%. 7 pacientes presentaron complicaciones: una perforación intestinal, dos perforaciones vesicales, una erosión de pared vaginal, tres incontinencias de urgencia de novo. En 6 casos persiste la incontinencia de esfuerzo. Al analizar la asociación entre las diversas variables y el éxito postquirúrgico, apreciamos que las variables con valor pronóstico fueron la edad y la obesidad $(\mathrm{P}<0.05)$ no observando relación con la técnica empleada.

Conclusiones: La existencia de múltiples abordajes quirúrgicos y un variado utillaje auxiliar permiten que cada cirujano pueda escoger aquel con el que esté más familiarizado y cómodo sin que, aparentemente, ello influya en los resultados.

Palabras clave: Incontinencia urinaria de esfuerzo femenina. Tratamiento. Sling suburetral. Estudio comparativo.

\section{ABSTRACT}

COMPARISON OF DIFERENTS SUBURETHRAL SLINGS FOR THE TREATMENT OF STRESS URINARY INCONTINENCE

Objectives: To compare the results of the different procedures of sling suburetral used in our service (TVT ${ }^{\circledR}$, Sparc ${ }^{\circledR}$, IVS $^{\circledR}$, Obtape ${ }^{\circledR}$ and Monarc ${ }^{\circledR}$ ) for the treatment of the female urinary incontinence.

Material and methods: 96 patients retrospective analysis affects of female urinary incontinence and subjected to different procedures of sling suburethral without tension. The half age: $56,1 \pm 10,7$ years. 75 patients were intervened by means of procedures retropubics (48 TVT, 17 Sparc, 10 IVS) and 21 with obturators (10 Obtape, 11 Monarc). The half time of pursuit: $26,3 \pm 12,7$ months $(29,4$ months for the methods retropubics and 11,7 for the obturators). The analyzed variables were: age, obesity, grade of previous incontinence, type of used procedure, time of pursuit, and previous surgical procedures. We compare the complications and the results obtained with the different ones technical.

Results: the cure percentage was of $95.7 \%$. 7 patients presented complications: one intestinal perforation, two bladders perforations, one erosion of vaginal wall, three incontinences of novo urgency. In 6 cases the stress incontinence persists. When analyzing the association between the diverse variables and the success, we appreciate that the variables with value presage were the age and the obesity $(\mathrm{P}<0.05)$ not observing relationship with the technique employeed.

Conclusions: The existence of multiple surgical approachs and a varied auxiliary materials allow each surgeon to choose that with the one that is more familiarized and comfortable without, seemingly, influence it in the results.

Keywords: Stress urinary incontinence. Treatment. Suburethral slings. Comparison. 
$\mathrm{A}^{\mathrm{r}}$ raíz de la comercialización y el éxito de la técnica TVT $^{\circledR}$ para el tratamiento de la incontinencia urinaria de esfuerzo (IUE) femenina (Ulmstein, 1996) ${ }^{1}$ otros productos, aparentemente similares, han ido apareciendo en los últimos años, creando en el urólogo la disyuntiva de cuál puede presentar las mayores ventajas.

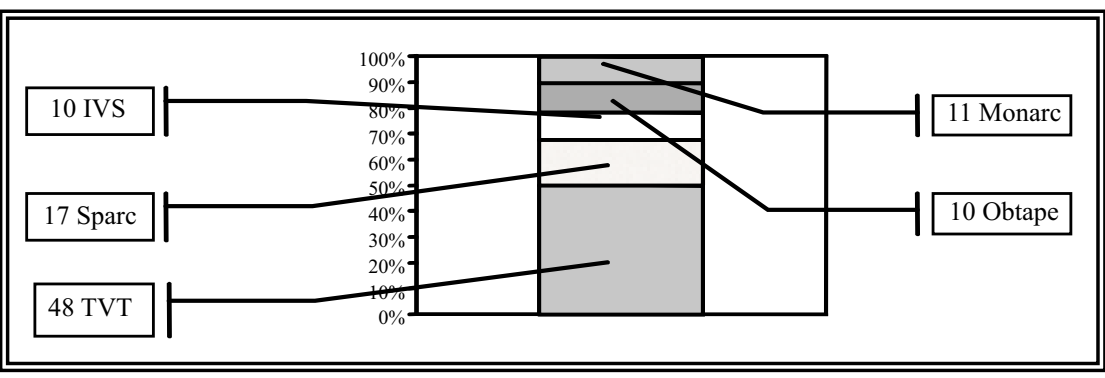

FIGURA 2. Diferentes procedimientos de slings utilizados, 75 suparpúbicos y 21 transobturatrices.
El fundamento de todas estas técnicas es el mismo: colocar un sling sin tensión debajo del 1/3 medio de la uretra, de forma que cierre la uretra en situaciones de stress. Con ello conseguimos un refuerzo "funcional" de los ligamentos pubouretrales y uretropélvico, asegurando una adecuada fijación del tercio medio de la uretra al pubis y simultáneamente reforzar la hamaca vaginal suburetral y su conexión con los músculos pubocoxígeos ${ }^{1}$.

Estos productos los podemos clasificar, según la vía de abordaje, en slings suprapúbicos (TVT ${ }^{\circledR}$, Sparc ${ }^{\circledR}$, IVS $^{\circledR}$ ) o transobturatrices (Obtape $^{\circledR}$, Monarc $^{\circledR}$ ), y según el entramado de la malla, tejido de mono-filamento en TVT $^{\circledR}$, Sparc $^{\circledR}$ y Monarc ${ }^{\circledR}$ y tejido de multi-filamento en IVS $^{\circledR}$ y Obtape ${ }^{\circledR}$ (Fig. 1), siendo el material base en todos ellos el polipropileno.

\section{PACIENTES Y MÉTODOS}

Se realiza un análisis retrospectivo de 96 pacientes afectas de IUE y sometidas a diferentes procedimientos de sling suburetral sin tensión en el Servicio de Urología del Hospital de Sagunto desde Febrero de 1999 a Junio del 2004. La edad media de las pacientes fue de 56,1 \pm 10,7 años. 75 pacientes se intervinieron mediante procedimientos suprapúbicos $\left(48\right.$ TVT $^{\circledR}, 17 \operatorname{Sparc}^{\circledR}, 10$ IVS $^{\circledR}$ ) y 21 con transobturatrices (10 Obtape ${ }^{\circledR}, 11$ Monarc $^{\circledR}$ ) (Fig. 2). El tiempo medio de seguimien- to fue de $26,3 \pm 12,7$ meses $(29,4$ meses para los métodos suprapúbicos y 11,7 para los transobturatrices). Historia gestacional: uno o más embarazos 94\% de las pacientes, nulíparas: 6\%. 32 pacientes $(35,1 \%)$ se consideraron obesas (>80 $\mathrm{Kg}$ ) 17 mujeres habian sido sometidas a intervenciones uro-ginecológicas previas: 6 por IUE (Técnica de Raz en 4 y Burch en dos), y en 11 pacientes se había realizado histerectomía previa por diversos motivos. El tiempo medio de intervención fue de 35 minutos para los procedimientos suprapúbicos (que siempre incluían cistoscopia) y 18 minutos para los transobturatrices (en los que se obvió). La estancia hospitalaria fue de 1,2 días (rango: 1-3) (Tabla 1). El grado de incontinencia pre-operatoria fue: grado I el 18\%, grado II el 52\% y grado III el 30\%.

En la evaluación preoperatoria de las pacientes consideramos: exploración física general y ginecológica, grado de IUE (Clasificación de Ingelmann) ${ }^{2}$ descartando inestabilidad del detrusor, presencia o no de prolapsos asociados (cistocele, rectocele, enterocele y prolapso uterino), ecografía abdómino-pélvica y urinocultivo.

La intervención se hizo siempre bajo raquianestesia, profilaxis antibiótica con Amoxicilinaclavulámico (o Tobramicina en casos de alergia a betalactámicos) no precisando profilaxis tromboembólica (a no ser que existieran factores de
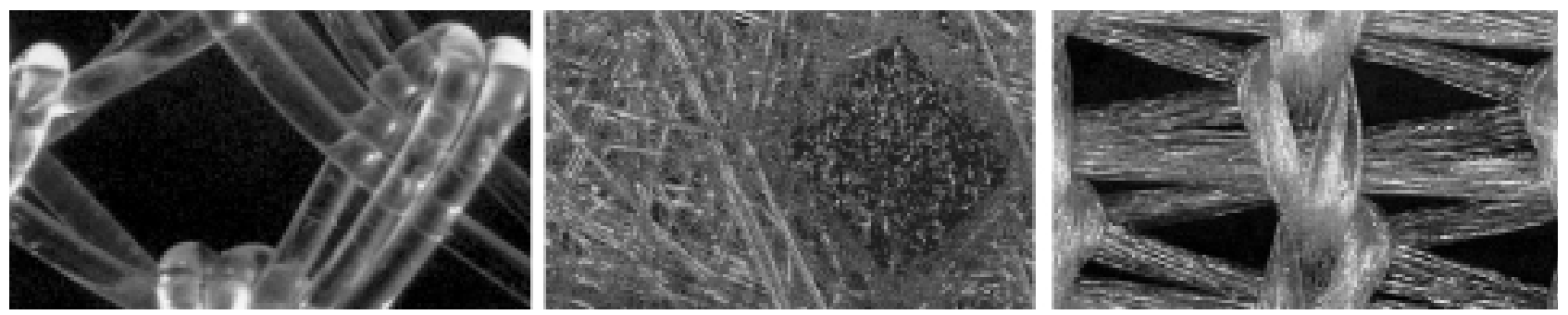

FIGURA 1. Diferentes tipos de mallas según el entramado. Cortesia de American Medical Systems. 
Tabla 1

Casuística

$\mathrm{n}=96$

Edad media: 56,1 \pm 10,7 años. Rango: 26-83

Historia gestacional: Embarazos 94\%. Partos: (1-4). Nulíparas: $6 \%$

Tiempo medio de seguimiento: $26,3 \pm 12,7$ meses

Procedimientos; suprapúbicos: 75 pacientes Transobturatrices: 21 pacientes

Obesidad (> $80 \mathrm{Kg}$ ): 32 pacientes

IQ previas: IUE: 6 (Raz en 4 y Burch en 2 Histerectomía previa: 11 pacientes

Tiempo quirúrgico: 35/18 min

Estancia hospitalaria: 1,2 días (rango: 1-3)

riesgo) dada la pronta movilización postoperatoria de las pacientes. La técnica utilizada fue la descrita para cada intervención concreta; en la Figura 3 esquematizamos el trayecto de las cintas para cada técnica y las estructuras vecinas.

Para valorar los resultados tuvimos en cuenta, por un lado la persistencia o no de la incontinencia y por otro lado la sensación de bienestar transmitida por la paciente. La realización prevista del test de la compresa ${ }^{3}$ se descartó por las dificultades de colaboración de la paciente y utilizamos el cuestionario de malestar urogenital de Uebersax (forma corta) ${ }^{4}$.

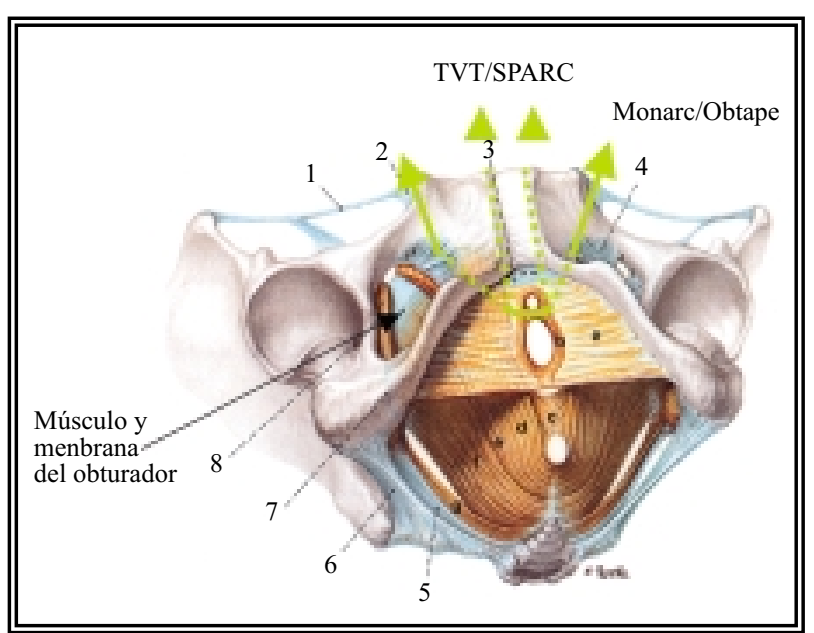

FIGURA 3. Trayecto de las cintas para cada técnica y las estructuras vecinas. Cortesia de American Medical Systems.
A las pacientes se les pasó, al mes y a los tres meses de la intervención, el cuestionario de malestar urogenital de Uebersax (forma corta) (Tabla 2). Se trata de un cuestionario estandarizado autoadministrado. Las pacientes respondieron a 6 preguntas referentes a su grado de malestar por: frecuencia miccional, escapes (por urgencia o por esfuerzos), pérdidas de gotas, dificultad para vaciar la vejiga y molestias en hipogastrio o área genital (incluyendo molestias coitales), respondiendo de 0 a $3(0=$ no le molesta nada; 3 = molestia grave).

La suma de la puntuación cruda se pasó a escala de 100 puntos, de forma que con una puntuación de menos de $30 \%$ se definió como una alto grado de satisfacción.

Se consideró criterios de curación: prueba de esfuerzo negativa y cuestionario de Uebersax $<30 \%$, criterio de mejoría: reducción significativa de la pérdida de orina y cuestionario de Uebersax 30\%-60\%, criterio de fracaso: resto de las pacientes.

En cuanto a las complicaciones, valoramos: lesiones iatrogénicas (vesical, intestinal, y uretral), hematoma, infección herida quirúrgica, erosión de la pared vaginal, residuo elevado, algias coitales, IU de urgencia "de novo", etc.

Confeccionamos una base de datos a partir de las variables consideradas (edad, historia gestacional, obesidad, intervenciones quirúrgicas previas del área pélvica, grado de incontinencia, tipo de procedimiento empleado, tiempo de seguimiento, complicaciones y resultados en cuanto a continencia). El programa empleado para su estu-

Tabla 2

Cuestionario de malestar urogenital de Uebersax (forma corta)

$\begin{array}{llll}0 & 1 & 2 & 3\end{array}$

Frecuencia

Pérdidas con esfuerzo

Pérdidas con la urgencia

Pérdida de gotas

Dificultad para orinar

Molestias hipogástricas, genitales y coitales 
dio estadístico fue el SPSS para Windows versión 10,0 de SPSS Inc. El análisis comprendió inicialmente el cálculo de distintos estadísticos de distribución. Para establecer la influencia de las variables analizadas en la aparición de complicaciones y en los resultados, se computó la frecuencia de sucesos en las distintas clases y se compararon entre sí con el test Chi-cuadrado mediante tablas de contingencia. En los casos en que una de las frecuencias era inferior a 5 se utilizó la prueba exacta de Fisher. Además, elaboramos curvas de Kaplan-Meier, aplicando el test de log-rank, para evidenciar si el tiempo de seguimiento influye o no en los resultados y si hay diferencias según la técnica quirúrgica empleada o las características previas de la paciente.

\section{RESULTADOS}

Continencia

Según los criterios anteriormente mencionados, 90 pacientes quedaron completamente curadas, 2 de ellas mejoraron significativamente y 4 fueron fracaso, lo cual supone un porcentaje de curación del 95.7\%. Dependiendo de la técnica utilizada, la persistencia de la IUE la encontramos en:

1. TVT $^{\circledR}$ en dos de casos de $48(4 \%)$,

2. Sparc $^{\circledR}$ en uno de 17 casos $(6 \%)$

3 . IVS $^{\circledR}$ en dos de 10 casos (20\%)

4. Monarc $^{\circledR}$ en cero de 11 casos $(0 \%)$

5 . Obtape ${ }^{\circledR}$ uno de 10 casos $(10 \%)$.

Llama la atención una paciente de 74 años sometida a múltiples técnicas: un Burch (en otro Centro) y posteriormente, en nuestro Servicio se realizaron un Raz, un Sling transobturatriz $\left(\right.$ Obtape $\left.^{\circledR}\right)$ y finalmente una técnica de sling suprapúbico $\left(\right.$ TVT $\left.^{\circledR}\right)$, persistiendo la incontinencia de la paciente; actualmente se le ha propuesto la colocación de un esfínter artificial.

\section{Complicaciones}

7 casos presentaron complicaciones $(7,2 \%)$ significativas: una perforación intestinal (al realizar una TVT $^{\circledR}$, que requirió laparotomía de urgencia), dos perforaciones vesicales, una erosión de pared vaginal, tres incontinencias de urgencia "de novo" con buena respuesta a anticolinérgicos sin ocasionar residuos elevados. Por otro lado, dos pacientes estuvieron inicialmente con residuos altos, resolviéndose espontáneamente tras una media de 16 días de cateterismo intermitente. La mayoría de las complicaciones corresponden a los primeros casos (curva de aprendizaje), en los que influyó decisivamente la dificultad de dar la tensión adecuada a la cinta (5 de 9 casos), ya que en buena parte va a depender de la experiencia del urólogo.

Asociación entre complicaciones y procedimientos retropúbicos/transobturatrices

1. TVT $^{\circledR}, 4$ de 48 (8\% de complicaciones): una perforación intestinal, una perforación vesical, una incontinencia de urgencia "de novo" y una erosión de pared vaginal.

2. $\operatorname{IVS}^{\circledR}$, una de 10 (10\% de complicaciones): una incontinencia de urgencia "de novo".

3. Sparc $^{\circledR}$, tres de $17(3.11 \%$ de complicaciones): una perforación vesical y dos urgencias "de novo".

4. Obtape ${ }^{\circledR}$, una de $10(10 \%$ de complicaciones): 1 caso de urgencia "de novo".

5. Monarc ${ }^{\circledR}$, no se apreciaron complicaciones ( $0 \%$ de complicaciones).

No hubo fístulas ni se apreció hemorragia significativa ni necesidad de transfusiones así como tampoco infección de la herida quirúrgica. Ninguna paciente se quejó de molestias coitales.

Las variables analizadas fueron: edad, obesidad, grado de incontinencia previa, tipo de procedimiento empleado, tiempo de seguimiento, y procedimientos quirúrgicos previos (IUE/Ginecológicos). Al analizar la asociación entre las diversas variables y el éxito postquirúrgico en cada uno de los diferentes procedimientos apreciamos que las variables con valor predictivo en el análisis univariante fueron la edad y la obesidad ( $P$ $<0,05$ ) (Tabla 3) no guardando relación con la técnica empleada y que, aunque parece existir mejores resultados con la TVT $^{\circledR}$ y el Monarc ${ }^{\circledR}$, las diferencias con el resto de las técnicas no son significativas.

Además se puso de manifiesto que el tiempo de seguimiento $(26,3 \pm 12,7$ meses $)$ no influye en los resultados ya que las pacientes que en la primera visita postcirugía permanecen secas, siguieron así el resto del seguimiento. 
Tabla 3

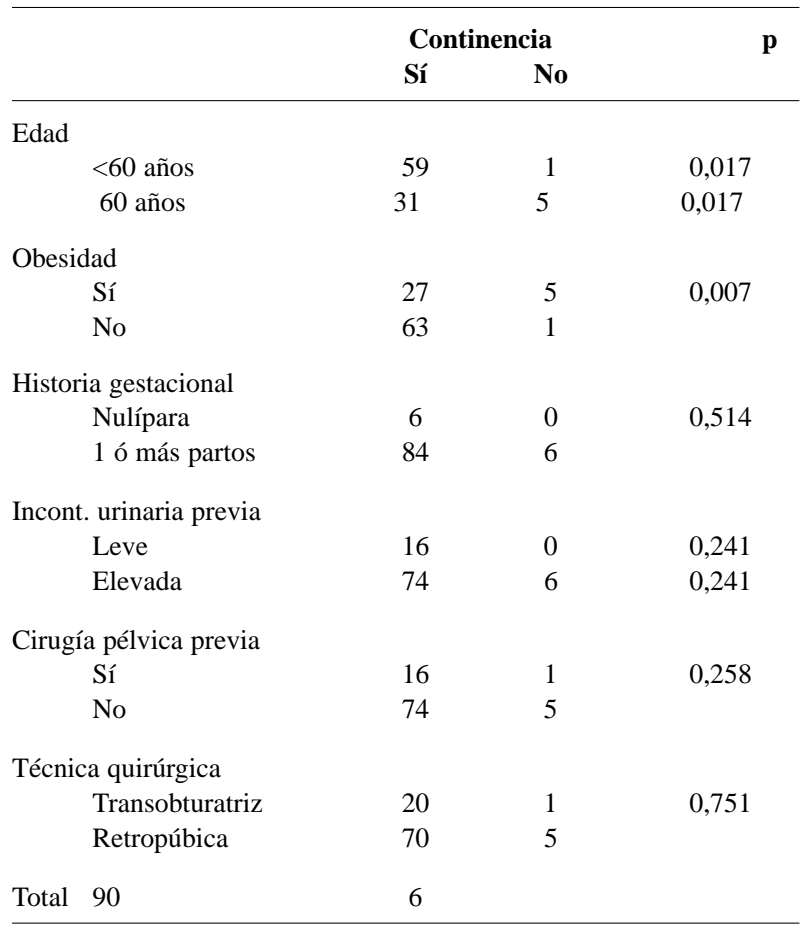

Tablas de contingencia en las que apreciamos que las variables con valor predictivo fueron la edad y la obesidad $(P<0,05)$.

\section{DISCUSION}

Las técnicas de sling se iniciaron a principio del siglo XX (se atribuye a Von Giordano ${ }^{5}$ en 1907 la primera descripción de un sistema de sling para el tratamiento de la incontinencia) pero no fue hasta el reconocimiento de la disfunción intrínseca del esfinter uretral, como entidad particular dentro de las causas de incontinencia, cuando su utilización se extendió. Inicialmente fueron consideradas, por la mayoría de los autores, como el tratamiento de elección en caso de disfunción esfinteriana primaria ${ }^{6-11}$.

Posteriormente autores como Appel1 ${ }^{9}$ y Blaivas $^{12}$ comenzaron a proponer los procedimientos tipo sling como el tratamiento de elección para todos los casos de incontinencia urinaria de esfuerzo, independientemente del tipo fisiopatológico basando su propuesta en los buenos resultados a largo plazo y a que estos procedimientos sirven para dar tanto soporte a la uretra hipermóvil, como buena coaptación a la uretra débil, tratando a todas las pacientes con una técnica efectiva en todos los tipos de incontinencia de esfuerzo.
Moir ${ }^{13}$ en 1968 introdujo la malla de polietileno (Mersilene ${ }^{\circledR}$ ) y Morgan ${ }^{14}$ en 1985 describe la utilización de polipropileno $\left(\right.$ Marlex $^{\circledR}$ o Prolene ${ }^{\circledR}$ ), el cual se incorpora adecuadamente en los tejidos, sin excesiva reacción fibrosa.

Sin embargo, los materiales sintéticos tienen significativamente mayor incidencia de complicaciones como infección, rechazo, erosión, fístulas, etc. La gran eclosión de la técnica se inició tras la comprensión de unos conceptos básicos que se han universalizado:

1. La necesidad de evitar la tensión en el sling

2. El uso más racional de los antibióticos, y

3. El uso de mejores materiales sintéticos en los últimos años, mejorando la adaptación de las cintas inorgánicas. Es el caso del politetrafluoretileno expandido (Gore-Tex $\left.{ }^{\circledR}\right)^{15}$ el silastic $^{16}$ y el mismo polipropileno (Prolene ${ }^{\circledR}$ ), para el cual autores como Ulmsten y cols ${ }^{1,17}$. describen una prácticamnete ausencia de rechazo y erosión.

El gran impulsor de las técnicas de sling sin tensión fue sin duda Ulmsten con la introducción en 1996 de la TVT ${ }^{\circledR 1}$ para el tratamiento de la incontinencia urinaria de esfuerzo femenina, fue a partir de entonces cuando otros productos, aparentemente similares, aparecieron y siguen apareciendo en los últimos años, creando en el urólogo la disyuntiva de cuál puede presentar las mayores ventajas.

El punto clave y más importante de este tipo de intervenciones es determinar cuánta tensión se le da al sling una vez colocado en tercio medio de uretra. Autores de experiencia como Mc Guire y cols ${ }^{7}$ puntualizan que esto es algo difícil de enseñar, pues es, en gran parte, subjetivo y se adquiere con la experiencia. Actualmente se acepta que el grado de tensión a la uretra prácticamente debe ser mínimo y para ello basta interponer una tijera entre la cinta y la uretra, lo cual no deja de ser un método completamente subjetivo y muy dependiente de la experiencia del urólogo.

Al realizar una minuciosa búsqueda bibliográfica, nos llama la atención el gran número de artículos que hacen referencia a cada una de las técnicas de sling sin tensión (más de 800 
artículos). Sin embargo, y a pesar de existir un apreciable tiempo de seguimiento en algunas de ellas, hemos encontrado muy pocos artículos que se refieran a estudios comparativos globales de los diferentes slings existentes en la actualidad. Recientemente, en esta misma revista, Galmés y Díaz ${ }^{18}$ realizan una interesante descripción de las características de las mallas existentes en el mercado y sus propiedades así como también de las diferentes vías de abordaje, no obstante dicha comunicación se realiza desde el punto de vista teórico sin aportar la experiencia de los autores, con lo cual no se aclara el interrogante que planteamos en este artículo: ¿existe alguna técnica que aporte claramente mejores resultados que las demás?.

La mayoría de autores, entre los que nos encontramos nosotros, aportan claros beneficios de las técnicas transobturatrices respecto a las suprapúbicas pero en aspectos que no hacen referencia a los resultados, como son el menor tiempo quirúrgico ${ }^{19}$. Otra clara ventaja de las técnicas transobturatrices sería el menor riesgo de lesiones yatrogénicas intestinal (pacientes sometidas a cirugía pelviana en la que el intestino pudiera estar muy próximo al pubis) y vesical ${ }^{20}$.

No obstante, y como dato importante a favor de las técnicas suprapúbicas, en nuestra experiencia (aunque solo ha sido una paciente), en caso de fracaso de una técnica transobturatriz optamos por una técnica suprapúbica como segunda intención.

Pensamos que todavía no existe una respuesta definitiva al interrogante que hemos planteado en este estudio: ¿existe alguna técnica que aporte claramente mejores resultados que las demás? No obstante, y siendo conscientes de nuestra limitada experiencia, creemos que, dada la existencia de múltiples abordajes quirúrgicos y un variado utillaje auxiliar, cada urólogo puede escoger aquella con la que esté más familiarizado y cómodo sin que, aparentemente, ello influya en los resultados, no obstante se requieren estudios randomizados a largo plazo y con mayor número de pacientes para validar esta suposición.

\section{REFERENCIAS}

1. Ulmsten U, Henriksson L, Johnson P, Varhos G. An ambulatory surgical procedure under local anaesthesia for treatment of female urinary incontinente. Int Urogynecol $\mathrm{J}$ 1996;7:81.

2. Ingelman-Sundberg, A. Urinary incontinence in women, excluding fictulas. Acta Obstet Gynecol Scand 1995;31: 266.

3. Mouristen L, Berlid G, Hertz J. Comparison of different methods for quantification of urinary leakage in incontinent women. Neurourol Urodyn 1989;8:579.

4. Uebersax, JS, Wyman, JF, Shumaker SA, Mcclish DK, Fanti JA and the Continence Program for Women Research Group. Short Forms to Assess Life Quality and Symptom Distress for Urinary Incontinence in Women: The Incontinence Impact Questionnaire and the Urogenital Distress Inventory. Neurourol Urodyn 1995; 14:131-139.

5. Goebell R. Zur operativen beseitigung der angebornen incontinentia vesicae. Zeitschr Gynäkol 1910;2:187.

6. Blaivas JG, Jacobs BZ. Pubovaginal fascial slings for the treatment of complicated stress urinary incontinence. J Urol 1991;145:1214.

7. Mc Guire EJ, O’Connell HE: Surgical treatment of intrinsic urethral disfunction: Slings. Urol Clin North Am 1995;22: 657.

8. Leach GE, Dmochowski RR, Appell RA, Blaivas JG, Hadley HR, Luber KM, et al: Female Stress Urinary Incontinence Clinical Guidelines Panel summary report on surgical management of female stress urinary incontinence. J Urol 1997;158:875.

9. Appell RA. Editorial: Primary slings for everyone with genuine stress incontinence? Int Urogynecol J 1998;9:249.

10. Summitt RL, Bent AE, Ostergard DR, et al: Suburethral sling procedure for genuine stress incontinence and low urethral closure pressure. A continued experience. Int Urogynecol J 1992;3:18.

11. Ghoniem GM, Shaaban A: Suburethral slings for the treatment of stress urinary incontinence. Int Urogynecol $\mathrm{J}$ 5:228, 1994.

12. Chaikin DC, Rosenthal J, Blaivas JG: Pubovaginal slings for all types of stress urinary incontinence: long-term analysis. J Urol 1998;160:1312.

13. Moir JC: The gauze hammock operation (a modified Aldridge sling procedure). J Obstet Gynaecol Br Commonw 1968; 75: 1 .

14. Morgan JE, Farrow GA, Stewart FE: The marlex sling operation for the treatment of recurrent stress urinary incontinence. A 16 year review. Am J Obstet Gynecol 1985;151: 224.

15. Horbach NS, Blanco JS, Ostergard DR, et al: A suburethral sling procedure with tetrafluoroethylene for the treatment of genuine stress incontinence in patients with low urethral closure pressure. Obstet Gynecol 1988;71:648.

16. Stanton SL, Brindley GS, Holmes DM: Silastic sling for urethral sphincter incompetence in women. Br J Obstet Gynaecol 1985;92:747. 
17. Ulmsten U, Hilton P, Ferrari A, et al: Tension-free Vaginal Tape procedure: a micro-invasive surgical technique for genuine stress incontinence. Int Urogynecol J 1997;8:1.

18. Galmés Belmonte I, Díaz Gómez E. ¿Son iguales todos los sistemas empleados para corregir la incontinencia urinaria mediante mallas libres de tensión? Actas Urol Esp 2004;28(7):487-496.

19. Ansquer Y, Marcollet A, Yazbeck C, Salomon L, Poncelet C, Thoury A, Dhainaut C, Madelenat P. The suburethral sling for female stress urinary incontinence: a retropubic or obturator approach? J Am Assoc Gynecol Laparosc. 2004 Aug; 11(3):353-8.
20. Kathleen C. Kobashi And Fred E. Govier. Perioperative complications: the first 140 polypropylene pubovaginal slings. J Urol 2003;170,1918-1921.

Dr. A. Borrell Palanca

Servicio de Urología.

Hospital de Sagunt

Avda. Ramón y Cajal s/n

46520 - Port de Sagunt (Valencia)

(Trabajo recibido 13 de enero 2005) 